\title{
DIFFERENCE BETWEEN PAINTING COLOURS \& TECHNICAL COLOURS
}

\author{
Dr. Pooja Gupta \\ Assistant. Prof. \& H.O.D.
}

Subharti Institute of Fine Arts \& Fashion Design S.V.S.U. Meerut

For a minute can we imagine the world around you without colors, how boring and unexciting life would be. Colors play a very important role in our life and it has been confirmed that our actions and responses are influenced by them.

Every color has a unique effect on individuals and arouses various responses. In modern time there are lots of confusions regarding colour theory or colour concepts as for a painting student colour theory is different and for computer designer a colour theory is extremely high configure. The whole concept is based on misconceptions which we face. To make it clear we need to study both concepts. So that we as an artist or student of painting knows the difference.

\section{PRESERVATIVE COLOUR}

When we studied colour concepts starting in elementary art and continuing in advanced art, printing, computer Science. There are lots of difference in the primary colours, complementary colours and the colour wheel that are taught to us in academic reference. The identification and classification of these differences and experiments that make clear these colour concepts as well as help us to understand the difference in practical colour or machine based colour.

As we all knows the three primary colours are red, yellow \& blue but according to chemical addition colours, "Computer based colours are those primary colors which can be defined by two characteristics as one is no combination of two primary colors can Produce a third primary color and the other one is Combining the three primary colors can produce a wider range of colors than using any other three colors." The primary preservative colors of light are generally as red, green, and Blue (RGB). Chemical addition color mixing can be discussed through the combinations as-

Red + Green $=$ Yellow

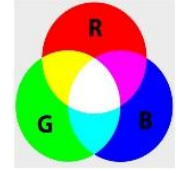

Green + Blue $=$ Cyan, Blue + Red $=$ Magenta

These relationships can be verified by us through examining how colors are made on a computer screen. For this we can says, the relationship of colour mixing to the color wheel can be madeYellow light is between red light and green light, because it is the result from the mixing of these colours of light. Same as cyan is between green and blue, and magenta is between blue and red. Students can also investigate with a magnifier how white is produced on a computer monitor the white background actually consists of small red, green, and blue lights (Red+ Green+ Blue = White). The concept of complementary colours also can be demonstrated using the colour wheel. Complementary colours of light are those two colours that when added together Produce white as Yellow and blue are complementary colours of light: 
Yellow + Blue $=($ Red + Green $)+$ Blue - White. Red and cyan are also complementary colours as are green and magenta. The colour wheel provides a nice way to view complementary colours they lie on opposite sides of the colour wheel.

\section{DEDUCTIVE COLOURS}

The primary deductive colours are cyan, magenta, and yellow. This term is well known to printers. But in art the primary colours of painting are red, yellow, and blue. This discrepancy is rarely addressed by art books; these misconception somehow primary deductive colours in physics or printing are different from those of painting.

White-Red=Cyan (Cyan pigment)
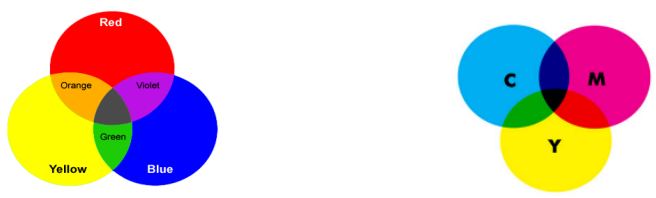

White-Green=Magenta (Magenta pigment)

White-Blue=Yellow (Yellow pigment)

These equations demonstrate that the colour of an object as cyan because it deducts a single primary colour of light, namely red, from the incident white light. The primary deductive colours are supposed that they deduct one of the primary colours of light from the occurrence white light. Deductive colour mixing occurs when two primary deductive coloured objects overlapped so that both objects deduct colours from the incident white light.

White-Red-Green=Blue (Cyan/magenta pigments)

White-Green-Blue=Red (Magenta/yellow pigments)

White-Blue-Red=Green (Yellow/cyan pigments)

For representation a cyan article overlapping magenta stuff (cyan/magenta). Now the cyan article soak up the red light from the incident white light, while the magenta article soak up the green light, so that the object become visible blue. A colour wheel that models is again simply generated and is shows that mixing cyan and magenta pigments creates blue. So in the colour wheel, blue is between cyan and magenta. In the same way, red is between magenta and yellow, and green is between yellow and cyan. The concept of complementary colours can again be demonstrated using the colour wheel. Complementary colours of Pigments are those two colours that, when it overlapped, it produces black. It is apparent that yellow and blue are complementary deductive colours, since yellow pigment soak ups blue light and blue pigment soak up red and green light: White - blue $-($ red + green $)=$ White $-($ red + green + blue $)=$ White - white $=$ black $($ Yellow $/$ blue Pigments)

Red and cyan are also complementary colours of Pigment as green and magenta. Again, complementary colours of Pigments lie on conflicting sides of the colour wheel. Except for the switching of the primary colours from red, green, blue to cyan, magenta, Yellow, the colour wheel for light (preservative colours) is the same as the colour wheel for pigments (deductive colours) shown.

\section{COLOR DELUSIONS}


Many factors contribute to continued misconceptions about colour. Art books may confuse preservative primaries with deductive primaries, or confuse mixing light with mixing pigments. But the most significant one is the incorrect colour wheel that most artists use and to which most students are introduced in basic school. This traditional colour wheel was used by Goethe as early as 1793. It is still used it seems that because it is sufficiently successful in describing colour mixing, and because of its extensive acceptance and perseverance in current teaching materials. The long history and variety of colour wheels and models are described in a number of publications. In the traditional colour wheel, which is used for painting, the primary colours are red, Yellow, and blue; these colours were chosen for their vivid clarity. It shows that mixing red and yellow produces orange, mixing blue and yellow produces green, and mixing red and blue Produces violet, as shown by this colour wheel. There are many ways to demonstrate that this colour wheel is incorrect. Complementary colours should lie across from each other on the colour wheel-in the traditional colour wheel, they don't' Red, yellow, and blue are affirmed to be the primary colours (of paint or pigment). One should remember that a Primary colour cannot be made from mixing two other primary colours. Yet, a red pigment can be made by mixing Yellow and magenta Pigments and blue can be produced by mixing cyan and magenta pigments. So red and blue cannot be Primary colours of Paint. Another complexity in demonstrating colour mixing is the lack of commercially available materials with correct optical properties. While color rows or films are available from a number of sellers, none of them are of the correct colour to easily demonstrate the desired colour mixing. For this reason, the creator developed films and other color mixing material is with the correct optical properties. Similar colored films can also be made by Printing large red, green, blue, cyan, magenta, and Yellow rectangular coloured areas on ink-jet transparency paper using $\mathrm{u}$, ink-jet printer.

\begin{tabular}{c|c}
\hline Hue & Initial \\
\hline Red $\left(0^{\circ}\right)$ & \\
\hline Yellow $\left(60^{\circ}\right)$ & \\
\hline Green $\left(120^{\circ}\right)$ & \\
\hline Cyan $\left(180^{\circ}\right)$ & \\
\hline Blue $\left(240^{\circ}\right)$ & \\
\hline Magenta $\left(300^{\circ}\right)$ & \\
\hline
\end{tabular}

The naming of colours also contributes to confusion. Cyan is often called blue, because it appears to be a light or sky blue. Cyan is often not used because the word is not in common usage. Magenta is often called red. Therefore, a printer may be using the colours of cyan, magenta, and Yellow, but will call them blue, red, and yellow. The sensitivity of the human eye to different colours also is a source of color misconceptions. We do not easily distinguish the color cyan from the color blue. Magenta and red are also seen alike colours.

\section{REFERENCE}

1 F. Gerritsen, Et'oltrtion in Color (Schiffer Publishing Ltd., West Chester, PA, 1988).

2 Confusing color the Physics Teacher, www.sci-edga.org/.../Confusing\%20Color\%20Concepts\%20Clarified\%2..

3 Mastering The art of painting, Vol-1H. Fullmann 